\title{
Gene expression profiling of Leishmania (Leishmania) donovani: overcoming technical variation and exploiting biological variation
}

\author{
S. DECUYPERE ${ }^{1,2}$, M. VANAERSCHOT ${ }^{1,3}$, S. RIJAL $^{4}$, V. YARDLEY ${ }^{5}$, L. MAES ${ }^{6}$, \\ S. DE DONCKER ${ }^{1}$, F. CHAPPUIS ${ }^{7}$ and J.-C. DUJARDIN ${ }^{1 *}$ \\ ${ }^{1}$ Department of Parasitology, Unit of Molecular Parasitology, Institute of Tropical Medicine, Nationalestraat 155, \\ Antwerp B-2000, Belgium \\ ${ }^{2}$ Division of Infection and Immunity, Institute of Biomedical and Life Sciences, University of Glasgow G12 8QQ, UK \\ ${ }^{3}$ Department of Biomedical Sciences, Faculty of Pharmaceutical, Biomedical and Veterinary Sciences, University of Antwerp, \\ Universiteitsplein 1, B-2610 Antwerp, Belgium \\ ${ }^{4}$ B.P. Koirala Institute of Health Sciences, Dharan, Nepal \\ ${ }^{5}$ Department of Infectious and Tropical Diseases, London School of Hygiene and Tropical Medicine, Keppel Street, \\ London WC1E $7 H T$, UK \\ ${ }^{6}$ Laboratory of Microbiology, Parasitology and Hygiene, Faculty of Pharmaceutical, Biomedical and Veterinary Sciences, \\ University of Antwerp, Universiteitsplein 1, B-2610 Antwerp, Belgium \\ ${ }^{7}$ Department of Community Medicine, Travel and Migration Medicine Unit, Hôpitaux Universitaires de Genève, \\ Rue Micheli-du-Crest 24, CH-1211, Geneva 14, Switzerland
}

(Received 25 June 2007; revised 25 August 2007; accepted 28 August 2007; first published online 12 October 2007)

\section{S U M MAR Y}

Gene expression profiling is increasingly used in the field of infectious diseases for characterization of host, pathogen and the nature of their interaction. The purpose of this study was to develop a robust, standardized method for comparative expression profiling and molecular characterization of Leishmania donovani clinical isolates. The limitations and possibilities associated with expression profiling in intracellular amastigotes and promastigotes were assessed through a series of comparative experiments in which technical and biological parameters were scrutinized. On a technical level, our results show that it is essential to use parasite harvesting procedures that involve minimal disturbance of the parasite's environment in order to 'freeze' gene expression levels instantly; this is particularly a delicate task for intracellular amastigotes and for specific 'sensory' genes. On the biological level, we demonstrate that gene expression levels fluctuate during in vitro development of both intracellular amastigotes and promastigotes. We chose to use expression-curves rather than single, specific, time-point measurements to capture this biological variation. Intracellular amastigote protocols need further refinement, but we describe a first generation tool for high-throughput comparative molecular characterization of patients' isolates, based on the changing expression profiles of promastigotes during in vitro differentiation.

Key words: Leishmania (Leishmania) donovani, standardization, comparative expression profiling isolates, variation gene expression.

\section{INTRODUCTION}

Gene expression profiling is becoming an important tool to study pathogenesis of infectious diseases as it allows molecular characterization of the cellular responses of host and pathogen (Chaussabel et al. 2003 ; Hromatka et al. 2005; McAleese et al. 2006). This type of study has become feasible since the introduction of 2 high-throughput expression profiling techniques: (i) microarrays, used for comparative analysis of thousands of genes in distinct RNA populations (Schena et al. 1995), and (ii) quantitative real-time PCR (Q-PCR), used for rapid

* Corresponding author: Institute of Tropical Medicine, Unit of Molecular Parasitology, Nationalestraat 155, B-2000 Antwerp, Belgium. Tel: +32 32476358 . Fax: + 32 3 2476359. E-mail: jcdujardin@itg.be simultaneous analysis of a specific set of genes in large sample collections (Heid et al. 1996; Vandesompele et al. 2002). Both these techniques are rapidly gaining popularity in Leishmania studies since the Leishmania genome sequences have become available (Duncan, 2004; Ivens et al. 2005 ; Peacock et al. 2007). However, the interpretation and standardization of gene expression studies in Leishmania might be more challenging in comparison to other eukaryotes due to the specific biology of this parasite.

Firstly, most protein-coding genes in Leishmania are transcribed polycistronically, and mature mRNA levels appear to be regulated primarily by posttranscriptional mechanisms such as mRNA stability (Boucher et al. 2002; Campbell et al. 2003; Clayton, 2002; Martinez-Calvillo et al. 2004). Therefore, differences in mRNA abundance are often not as pronounced as in other organisms where expression 
is regulated at the level of transcription initiation (Saxena et al. 2003; Akopyants et al. 2004; Almeida et al. 2004; Duncan et al. 2004). The detection of such small differences is technically possible but demands sensitive and robust assays with strict measures to ensure reproducibility and quality control.

Secondly, Leishmania continuously changes lifeform throughout its life-cycle (in the vector from non-infective procyclic to infective metacyclic promastigotes; intracellular amastigotes in the host). This process of differentiation is associated with variation in gene expression, which in turn seems to be triggered by the changing external environment of the parasite (Shapira et al. 1988; Duncan et al. 2001 ; Saxena et al. 2007). The differentiation process can be mimicked in vitro by changing medium/ environment. Promastigotes grow from early log phase (procyclic forms) to stationary phase (metacyclic forms) in response to nutrient depletion and acidification of culture medium over 7-8 days (Sacks, 1989; Bates and Tetley, 1993; Zakai et al. 1998). Intracellular amastigotes initiate transformation to promastigotes within $1 \mathrm{~h}$ after release from the host cell (Fong and Chang, 1981). Previous studies have proven that gene expression/protein profiles vary considerably during the above-described in vitro differentiation (Akopyants et al. 2004; Holzer et al. 2006; McNicoll et al. 2006; Cohen-Freue et al. 2007; Saxena et al. 2007). Consequently, when comparing different Leishmania strains using gene expression profiles, it is of major importance to ensure that the studied parasites from the various strains (i) are in a similar in vitro differentiation stage and (ii) are obtained with in vitro manipulation techniques involving minimal disturbance of the parasite's environment to minimize impact on expression profile.

In this study we specifically wanted to explore the possibilities, requirements and limitations of gene expression profiling when used for molecular comparison of multiple Leishmania isolates from patients. We verified the impact of changing biological (differentiation stage) and technical (in vitro manipulation methods) parameters on expression levels of genes encoding for proteins with diverse functions. The results could be translated into guidelines for design of reliable comparative gene expression assays useful for high-throughput characterization of patient's isolates. Furthermore, this study also highlights some issues that might help the interpretation and comparison of other studies on Leishmania gene expression.

MATERIALS AND METHODS

\section{Parasites}

The L. (L.) donovani isolates BPK206/0, BPK091/0, BPK087/0, and BPK190/0 were obtained from bone-marrow aspirates from confirmed visceral leishmaniasis patients recruited at the B.P. Koirala Institute of Health Sciences, Dharan, Nepal. Leishmania species identification was done by PCRRFLP analysis of cysteine proteinase B as reported elsewhere (Tintaya et al. 2004). The uncloned isolates were tested as intracellular amastigotes for their in vitro antimonial susceptibility within 8 in vitro passages after isolation from patients, as described before (Rijal et al. 2007). Isolates BPK206/0 and BPK091/0 were characterized as $\mathrm{SbV}$ sensitive, and BPK087/0 and BPK190/0 as SbV resistant. These isolates were used here for gene expression analysis within a maximum of 15 in vitro passages after isolation from patients.

\section{In vitro promastigote generation}

Two protocols were used in this study for the generation of promastigotes. (1) Rapid growth on blood agar. Promastigotes were grown in Tobie's blood agar medium (Tobie et al. 1950) with a saline overlay at $26^{\circ} \mathrm{C}$. This rich medium supports promastigote growth and differentiation from procyclic to metacyclic stage in 3-4 days. The resulting cultures were harvested when the stationary phase was reached as determined by microscopical evaluation of morphology and parasite density. The overlay of the resulting culture was removed and washed 3 times with PBS to remove contaminating material originating from the blood agar. (2) Controlled growth on Eagle's medium. Promastigotes were grown on modified Eagle's medium (Mottram et al. 1992) (Invitrogen) supplemented with $20 \%$ (v/v) heat-inactivated foetal calf serum (PAA Laboratories $\mathrm{GmbH})$, pH $7 \cdot 5$, at $26^{\circ} \mathrm{C}$ over $7-8$ days. The cultures were initiated by inoculating metacyclic parasites (at day 3-4 stationary phase) in $5 \mathrm{ml}$ of culture medium at a final concentration of $5 \times 10^{5}$ parasites/ $\mathrm{ml}$. The parasite density was determined every $24 \mathrm{~h}$ using disposable count chambers Uriglass (Menarini diagnostics) to follow up the growth and differentiation profile. Parasites were harvested at different time-points of the growth curves (specific timepoints per experiment are specified in the text) by centrifugation and the resulting pellet was washed once with PBS. An identical batch of culture medium and foetal calf serum was used for all promastigote cultures in this study, to minimize variation in culture conditions.

\section{In vitro intracellular amastigote generation}

Murine peritoneal macrophages were infected with promastigotes at the third day stationary phase (as determined by the concomitant growth curve, see above 'controlled growth Eagle's medium') at a ratio of 7 promastigotes to 1 macrophage as described elsewhere (Decuypere et al. 2005); for all experiments, $>80 \%$ of macrophages were infected at 
$24 \mathrm{~h}$. The harvest of the in vitro-generated amastigotes was done at several time-points post-infection according to one of the following 2 protocols. (1) Purification of amastigotes by SDS lysis of macrophages. The adherent, infected macrophages were washed with ice-cold PBS to remove extracellular promastigotes. Three $\mathrm{ml}$ of $0 \cdot 0125 \% \mathrm{SDS} / \mathrm{PBS}$ were added to each $25 \mathrm{~cm}^{2}$ culture flask, and gently agitated until macrophages lifted and started to disintegrate. After dilution with PBS and mixing, the contents of each flask were aspirated through a $25 \mathrm{G}$ needle, causing further shearing of the macrophages, and transferred to a $50 \mathrm{ml}$ tube for centrifugation and 2 washing steps in PBS to remove macrophage debris. (2) Co-harvest of amastigotes/ macrophages. The adherent, infected macrophages were washed with RPMI 1640 (pre-heated to $37^{\circ} \mathrm{C}$ ) to remove residual promastigote debris. The infected macrophages were subsequently dissociated from the culture vessels by treatment with TrypLE Select (Invitrogen) for $9 \mathrm{~min}$ at $37^{\circ} \mathrm{C}$ as recommended by the manufacturer. The resulting cell suspension was immediately diluted 15 times with either RPMI 1640 or the buffer PSGEMKA (specified per experiment in the text). The buffer PSGEMKA was described to stall transformation of free amastigotes to promastigotes (Hart et al. 1981). The surface of the culture flask was carefully scraped with a cell scraper to lift loosely attached macrophages. The resulting suspension was transferred to a $50 \mathrm{ml}$ tube for centrifugation and washed once using the same buffer as in the previous step. The whole procedure was carefully timed and finished in 30-35 min.

\section{$R N A$ isolation/analysis and real-time quantitative PCR}

After the last washing step all harvested cultures were immediately disrupted in RNAqueous Lysis/ Binding solution (Ambion) and frozen at $-80{ }^{\circ} \mathrm{C}$ until RNA extraction. Total RNA was extracted, treated and analysed as described before (Decuypere et al. 2005). Reverse transcription of total RNA and expression profiling of the genes listed in Table 1 were performed as described elsewhere (Decuypere et al. 2005). All primers specifically amplify Leishmania cDNA as confirmed by quantitative assays on artificial Leishmania:macrophage cDNA mixtures with variable ratios between $1: 1$ and $1: 30$. Normalization of gene expression was done by determining the 4 most stable expressed genes from the set of 13 tested genes (Table 1), as described elsewhere (Vandesompele et al. 2002). Multiple Q-PCR runs of one particular experiment were calibrated (based on 3 samples included in each run for that purpose), organized and processed using qBase, a VBA application for MS Excel designed for the management and automated analysis of real-time quantitative PCR data (Hellemans et al. 2007).
Quantitative data of independent experiments were compared by determining the expression variation measure as described below.

\section{Definition expression variation measure $(E V M)$ and technical variation threshold (TVT)}

The expression variation measure (EVM) assesses a sample's variation in gene expression levels between repeated independent experiments. The mathematical background of EVM is outlined below.

If for 2 samples $\mathrm{A}$ and $\mathrm{B}$, the relative expression level of a particular gene $\mathrm{G}$ is determined in 2 independent experiments $\mathrm{X}$ and $\mathrm{Y}$, then the results of the two experiments can only be compared indirectly by considering the ratios:

for exp. X: $\frac{a_{x}}{b_{x}}$ for exp. Y: $\frac{a_{y}}{b_{y}}$

$a_{x / y}=$ sample A's relative expression level of gene $G$ in exp. $\mathrm{x} / \mathrm{y}$

$b_{x / y}=$ sample B's relative expression level of gene $G$ in $\exp . \mathrm{x} / \mathrm{y}$

The difference between these two ratios indicates that gene $G$ expression levels varied between the two experiments, either in one sample, or in both samples to a different degree. The more the two ratios differ, or, expressed in mathematical terms, the higher the value of the expression

st. dev. $\left\{\log _{2}\left(\frac{a_{x}}{b_{x}}\right), \quad \log _{2}\left(\frac{a_{y}}{b_{y}}\right)\right\} ;$

the more gene $G$ expression levels varied between the independent experiments for samples A/B.

Interpolation in function (1) of all 'gene $G$ '-ratios involving (i) sample A and (ii) every other sample included in experiments $\mathrm{X}$ and $\mathrm{Y}$ results in a function that summarizes sample A's individual interexperimental variation in gene $\mathrm{G}$ expression level. For sample A (of $\mathrm{N}$ samples in experiments $\mathrm{X}$ and $\mathrm{Y}$ ), this function is mathematically represented by the array:

$\forall \mathrm{A}, \mathrm{K} \in[1, \mathrm{~N}]$ and $\mathrm{A} \neq \mathrm{K}:$

$\left\{\text { st. dev. }\left\{\log _{2}\left(\frac{\mathrm{a}_{\mathrm{x}}}{\mathrm{k}_{\mathrm{x}}}\right), \quad \log _{2}\left(\frac{\mathrm{a}_{\mathrm{y}}}{\mathrm{k}_{\mathrm{y}}}\right)\right\}\right\}_{\mathrm{k}=1 \rightarrow \mathrm{n}}$

$a_{x / y}=$ sample A's relative expression level of gene $G$ in $\exp . \mathrm{x} / \mathrm{y}$

$\mathrm{k}_{\mathrm{x} / \mathrm{y}}=$ sample $\mathrm{K}$ 's relative expression level of gene $\mathrm{G}$ in $\exp . \mathrm{x} / \mathrm{y}$

$\mathrm{n}_{\mathrm{x} / \mathrm{y}}=$ sample N's relative expression level of gene $\mathrm{G}$ in $\exp . \mathrm{x} / \mathrm{y}$

The average of this array was defined in this study as the EVM of sample A for gene $\mathrm{G}$ between experiment $\mathrm{X}$ and $\mathrm{Y}$. 
Table 1. Overview primer design and PCR conditions for quantitative PCR

\begin{tabular}{|c|c|c|c|c|c|}
\hline Gene & Coding for & Function/Relevance & Sequence & Forward/Reverse Primer ${ }^{\mathrm{a}}$ & Fin. conc. \\
\hline AQP1 & aquaglyceroporin 1 & $\begin{array}{l}\text { integrated membrane channel for transport } \\
\text { water and small solutes }\end{array}$ & LinJ 31.0020 & 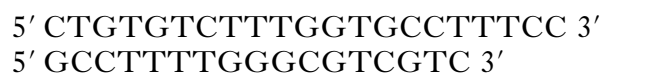 & $400 \mathrm{nM}$ \\
\hline MRPA & multidrug resistance protein $\mathrm{A}$ & $\begin{array}{l}\text { intracellular ABC transporter, presumably } \\
\text { involved in sequestration metal-thiol conjugates }\end{array}$ & LinJ 23.0290 & $\begin{array}{l}\text { 5' CGAAAGTTGAGCAGGAGACG 3' } \\
\text { 5' AATCCCCAAGCAGCCAGAC 3' }\end{array}$ & $300 \mathrm{nM}$ \\
\hline PRP1 & pentamidine resistance protein 1 & $\begin{array}{l}\text { intracellular } \mathrm{ABC} \text { transporter involved in } \\
\text { pentamidine resistance }\end{array}$ & LinJ 31.1810 & 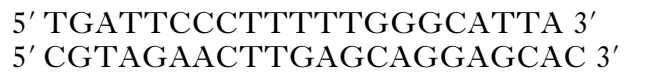 & $400 \mathrm{nM}$ \\
\hline ACR2 & arsenate reductase 2 & $\begin{array}{l}\text { protein tyrosine phosphatase activity and metal } \\
\text { reductase activity in presence of glutathione }\end{array}$ & LinJ 32.3240 & $\begin{array}{l}5^{\prime} \text { GCCCAGTCGCTCATACGG 3' } \\
5^{\prime} \text { AGAACGCCTCCCACCCAC } 3^{\prime}\end{array}$ & $400 \mathrm{nM}$ \\
\hline TDR1 & thiol dependent reductase 1 & homologue of glutathione S-transferase & LinJ 33.0270 & 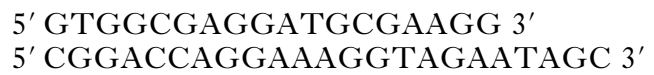 & $500 \mathrm{nM}$ \\
\hline CS & cysteine synthase & $\begin{array}{l}\text { enzyme for de novo cysteine biosynthesis } \\
\text { from serine }\end{array}$ & LinJ36.3190 & $\begin{array}{l}\text { 5' GTCTTGGCGGTTCAGTTCG 3' } \\
5^{\prime} \text { GACATTGTGGTTCGTCTGCTC } 3^{\prime}\end{array}$ & $500 \mathrm{nM}$ \\
\hline CBS & cystathione $\beta$-synthase & $\begin{array}{l}\text { enzyme in cysteine synthesis from } \\
\text { homocysteine via trans-sulphuration pathway }\end{array}$ & LinJ17.0280 & $\begin{array}{l}\text { 5' CGCCGATGTCAACTGGATG 3' } \\
5^{\prime} \text { GCTCCTTCTTCAGCGTGTCG 3' }\end{array}$ & $300 \mathrm{nM}$ \\
\hline SAT & serine acetyltransferase & $\begin{array}{l}\text { enzyme in de novo cysteine biosynthesis } \\
\text { from serine }\end{array}$ & LinJ 34.2490 & $\begin{array}{l}\text { 5' CCCGTATGCTGACAGAGTTGG 3' } \\
\text { 5' GCCGTGGTGAATGAAGAAGTG 3' }^{\prime}\end{array}$ & $400 \mathrm{nM}$ \\
\hline GCS & $\gamma$-glutamylcysteine synthase & key enzyme in glutathione biosynthesis & AY371486 ${ }^{\mathrm{b}}$ & $\begin{array}{l}5^{\prime} \text { TTTGCGTCCTGGTGCCTC } 3^{\prime} \\
5^{\prime} \text { TCAATGTTTAGTTGGGGGTCC } 3^{\prime}\end{array}$ & $500 \mathrm{nM}$ \\
\hline ODC & ornithine decarboxylase & key enzyme in spermidine biosynthesis & M81192 ${ }^{\mathrm{b}}$ & $\begin{array}{l}5^{\prime} \text { ATCCACCTCCAACCCGC } 3^{\prime} \\
5^{\prime} \text { TCCGCAACAGCAACAACAG } 3^{\prime}\end{array}$ & $500 \mathrm{nM}$ \\
\hline TR & trypanothione reductase & $\begin{array}{l}\text { central oxidoreductase for maintenance cellular } \\
\text { redox potential }\end{array}$ & $\mathrm{Z} 23135^{\mathrm{b}}$ & $\begin{array}{l}\text { 5' GGCGAGGTTCTGGGTGTTC 3' } \\
\text { 5' GACTCCGATGGTGCTGTGG 3' }\end{array}$ & $300 \mathrm{nM}$ \\
\hline MST & $\begin{array}{l}\text { mercapto-pyruvaat } \\
\text { sulfurtransferase }\end{array}$ & $\begin{array}{l}\text { assimilatory sulphur reduction and putative role } \\
\text { in detoxification ROS via thioredoxin oxidation }\end{array}$ & LinJ05.0980 & 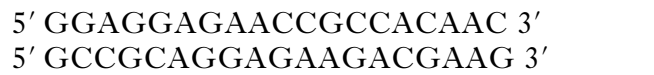 & $500 \mathrm{nM}$ \\
\hline S8 & ribosomal protein $\mathrm{S} 8$ & structural constituent of large subunit ribosome & LinJ 24.1470 & $\begin{array}{l}\text { 5' GCAGACAGGAAGACCACCAAG 3' } \\
5^{\prime} \text { AGCGGCGTGGACGGACT } 3^{\prime}\end{array}$ & $400 \mathrm{nM}$ \\
\hline
\end{tabular}

$\mathrm{a}=$ all primers were Leishmania specific $; \mathrm{b}=$ GenBank annotation, all other sequence no. as annotated in L. (L.) infantum GeneDB. 
Part of a sample's inter-experimental variation comprised in EVM will be caused by technical variation between the experiments. This technical variation depends on RT, PCR efficiency, PCR sensitivity, etc and is thus characteristic for each particular gene expression assay. The technical variation for each gene expression assay was assessed here by testing 8 samples in 4 technical repeated (=using identical RNA source) experiments. The average of the resulting 8 EVMs/gene reflects the inter-experimental technical variation for each gene expression assay. Or, extrapolating according to the Gaussian distribution, $95 \%$ of technically repeated samples would have an EVM smaller than:

$\frac{\sum_{\mathrm{i}=1}^{8} \mathrm{EVMi}}{8}+1.96 \times$ st. $\operatorname{dev}\left\{\mathrm{EVMi}_{\mathrm{i}=\mathbf{1} \rightarrow \mathbf{8}}=\mathrm{TVT}_{\mathrm{G}}\right.$

$\mathrm{EVMi}=\mathrm{EVM}$ of sample $\mathrm{i}$ between 4 technical repeated assays for gene $\mathrm{G}$;

which was here defined as technical variation threshold (TVT) of the expression assay of gene G.

\section{Interpretation EVM and TVT}

If EVM of sample A for gene $\mathrm{G}$ is lower than TVT of the expression assay for that gene $G$, the observed variation in expression level between experiments does not exceed the expected technical variation, and it is likely that sample A did not vary on the biological level for gene $\mathrm{G}$ expression between the independent experiments.

If EVM of sample A for gene $G$ is higher than TVT of the expression assay for that gene $G$, the observed variation in expression level between experiments is higher than expected by technical variation, and it is likely that sample A varied on the biological level for gene $G$ expression between the independent experiments.

We must emphasize, however, that EVM/TVT is a rough estimation of the biological expression variation in repeated independent experiments. As, first of all, TVT is only based on a limited number (4) of technical repeats and thus only an approximation of the actual technical variation of a gene expression assay; secondly, an EVM below TVT is no guarantee that there was only technical variation; as the biological variation between 2 particular experiments could be lower than TVT as well; and thirdly, EVM does not take into account the variability of the normalization factor, which, characteristic for it's stable nature, is minimal and constant through all samples.

\section{RESULTS}

\section{Exploring the limitations of Leishmania gene expression profiling: influence of cultivation and harvesting protocols}

The first aim of this study was to explore the impact of variable in vitro manipulation techniques on gene expression in both promastigotes and amastigotes. We therefore performed 2 independent experiments for expression profiling of 8 genes in promastigotes and amastigotes of $4 L$. (L.) donovani strains. The 2 experiments, referred to as Experiments $\mathrm{A}$ and $\mathrm{B}$, differed in protocols for parasite culturing and harvesting (details are listed in Fig. 1A). The 8 'target' genes encode proteins with different functions (Table 1), including (i) transport (AQP1, MRPA), (ii) redox metabolism (GCS, ODC, TR), (iii) cellular reduction (TDR1, ACR2) and (iv) ribosomal function (S8). This allowed us to verify if various cellular functions are affected to different extents at gene expression level by varying experimental conditions.

We introduced the expression variation measure (EVM) and technical variation threshold (TVT) to assess a sample's inter-experimental biological variation at the level of gene expression (see the Materials and Methods section). The EVMs of all samples based on the expression levels obtained in Experiments A and B are graphically depicted in Fig. 1B. Firstly, the graph clearly indicates that there was biological variation between the two experiments; with EVMs higher than TVT for several amastigote and promastigote samples. Secondly, the biological variation affected the expression of the various genes to a different extent, exemplified by the profiles of AQP1, GCS and TDR1. (i) For AQP1, the expression levels of all amastigote and promastigote samples showed biological variation between the two experiments (all EVMs $>$ TVT). The actual AQP1 expression profiles of the 4 isolates (promastigotes and amastigotes) obtained in Experiments A and B are depicted in Fig. 1C and demonstrate the extent of differences between the two experiments. (ii) The expression profiles of GCS showed a similar problem, but only on the level of amastigotes (all amastigote EVMs > TVT); promastigote results were similar in both experiments (all promastigote EVMs <TV'T). Fig. 1D graphically illustrates the similar promastigote and dissimilar amastigote GCS expression profiles of Experiments A and B. (iii) In contrast, the expression of gene TDR1 in both promastigotes and amastigotes was not affected by the variation between the two experiments, resulting in similar expression profiles (all EVMs < TVT; Fig. 1E).

\section{Identifying biological and technical sources of variation gene expression in Leishmania}

Intracellular amastigotes. We further focused on optimization and standardization of the simple and rapid harvest method used in Experiment $B$, a protocol which also seems favourable from a biological point of view (minimal disturbance of intracellular parasite environment). We verified the influence of the following 3 parameters on expression of 13 genes 


\section{(A) Differences in vitro manipulation between Experiments A and B}

\begin{tabular}{|c|c|c|}
\hline promastigotes & experiment A & experiment B \\
\hline culture medium & $\begin{array}{l}\text { Tobie's blood agar } \\
\text { \& saline overlay }\end{array}$ & $\begin{array}{l}\text { Eagle's medium } \\
+20 \% \text { HIFCS }\end{array}$ \\
\hline time of harvest & approx. start stationary phase & $\begin{array}{l}3 \text { days stationary phase as } \\
\text { determined by growth curve }\end{array}$ \\
\hline amastigotes & experiment $\mathrm{A}$ & experiment B \\
\hline harvest technique & $\begin{array}{l}\text { SDS/needle purification } \\
\text { from } \mathrm{m \Phi}\end{array}$ & $\begin{array}{l}\text { co-harvest macrophage in } \\
\text { RPMI } 1640\end{array}$ \\
\hline time-span harvest & $1-4 \mathrm{hrs}$ & 30-35 min \\
\hline
\end{tabular}

(C) AQP1

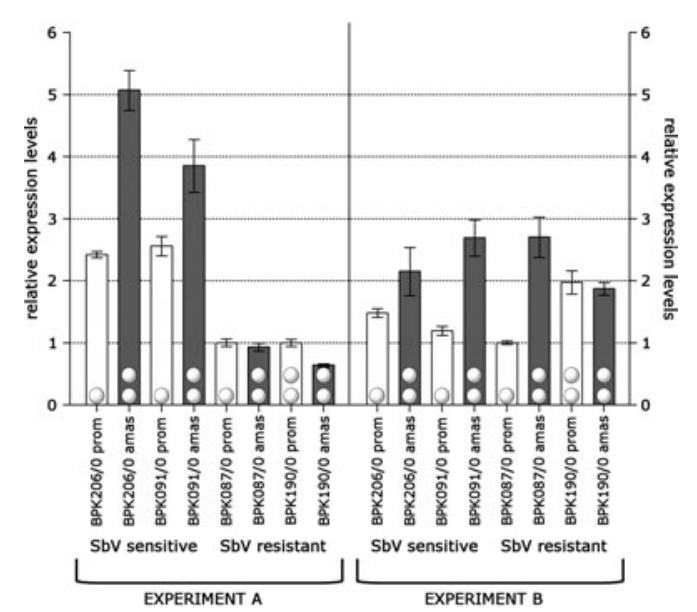

(B) EVMs between Experiments A and B for 8 genes in 4 L. (L.) donovani isolates (each isolate presented by 1 rectangle for prom. and 1 circle for amas.)

Fig. 1. Summary of gene expression analysis of Leishmania (L.) donovani promastigotes and intracellular amastigotes using different in vitro manipulation techniques.

$(\mathrm{C}-\mathrm{D})$ Comparison expression levels $( \pm$ s.D.) of the same strains (promastigotes $=$ white bars, amastigotes = grey bars) in independent Experiments A and B; white buttons inside bars indicate sample's EVM, with 1 button $=>$ than TVT but $<$ than $2 \times$ TVT; 2 buttons $=\geqslant 2 \times$ TVT; expression levels/gene rescaled to the respective sample with lowest EVM between Exp. A and Exp. B. 
(Table 1 ) in $L$. (L.) donovani in vitro infected in peritoneal macrophages. (i) Time post-infection macrophages $(24 \mathrm{~h}, 48 \mathrm{~h}, 72 \mathrm{~h}, 120 \mathrm{~h})$. Upon infection of macrophages, metacyclic promastigotes gradually transform into amastigotes; the rate of this differentiation (and adjusting gene expression) possibly varies between strains. We followed the biological changes in gene expression during the first $120 \mathrm{~h}$ post-infection (all harvests in PSGEMKA). (ii) Buffer used during harvest (RPMI 1640 or PSGEMKA). Manipulation of infected macrophages inevitably involves some shearing, which results in release of amastigotes (observed by microscopy). The buffer used during harvest could influence gene expression of those released amastigotes and perhaps of the intracellular amastigotes too. We compared 2 buffers in parallel harvests at $48 \mathrm{~h}$ post-infection: RPMI 1640, which is the basis of the medium (commercially available) used for macrophage cultures, and PSGEMKA (home-made) which was reported to preserve the biological status of amastigotes (Hart et al. 1981). (iii) Harvest time-span: the procedure is normally finished in 30-35 min; but variation in manipulation time might affect expression profiles. We verified the influence of harvest time-span by comparing expression profiles of 2 parallel harvests at $48 \mathrm{~h}$ post-infection including one 'short' harvest (the normal 30-35 min) and and 'long' harvest (normal harvest with additional 45 min incubation at $4{ }^{\circ} \mathrm{C}$ ). This comparison was done for both buffer systems.

All conditions were tested on a single large-scale infection (2 flasks/condition), thus ensuring uniform quality of in vitro infections in the various tested conditions. This experiment was performed in 2 strains, BPK206/0 ( $\mathrm{SbV}$ sensitive) and BPK190/0 ( $\mathrm{SbV}$ resistant), and the comparative expression profiles were determined in 1 quantitative experiment. As expected, the different parameters influenced the expression of the 13 genes to a variable extent. (i) Nine out of 13 genes showed a variable expression at the different biological time-points and (ii) 6 out of 13 genes had variable expression levels at $48 \mathrm{~h}$ post-infection when either different buffers or different harvest time-spans were used. Overall, we identified 3 types of profiles that are represented by AQP1, GCS and TDR1 (Fig. 2).

(i) AQP1 expression was found to be very unstable, which agrees with the results discussed in the previous section. The expression levels of BPK190/0 differed significantly between the different biological time-points (24-120 h post-infection) and between the different technical conditions (PSGEMKA vs RPMI 1640, two harvest time-spans, see Fig. 2A). We could not even reproduce the AQP1 expression profile of BPK190/0 in 2 biologically repeated harvests with identical conditions (data not shown).

(ii) The profile of GCS also showed considerable variation at the different biological time-points, particularly for BPK190/0; but all the technical conditions tested at $48 \mathrm{~h}$ post-infection gave reproducible results (Fig. 2B).

(iii) The expression of TDR1 was similar at all biological time-points and was not affected by varying technical parameters, which tallies with the results of the previous section (Fig. 2C).

Furthermore, the variability of expression for different biological and technical conditions seems to be strain dependent. Fig. 2 shows a contrasting picture of a 'stable' BPK206/0 profile versus a 'variable' BPK190/0 profile. This difference in stability of gene expression levels might reflect a difference in cellular flexibility. BPK190/0 and BPK206/0 are genetically closely related (Laurent et al. 2007), but differ in many phenotypic aspects, including in vitro $\mathrm{SbV}$ and SbIII susceptibility (Rijal et al. 2007), which could indeed be linked to differential cellular response ability. This inter-strain variation highlights the importance of choosing representative reference strains when standardizing such protocols.

Promastigotes. The first section demonstrated that the expression profiles of promastigotes also varied between Experiments A and B (Fig. 1). We suspected that these differences were due to unsynchronized harvesting during the gradual differentiation process of cultured promastigotes. Consequently, we chose the protocol 'controlled growth on Eagle's medium' (see the Materials and Methods section) for further standardization of promastigote expression profiling. Promastigotes were harvested every $24 \mathrm{~h}$ for 8 days for the 2 strains BPK206/0 and BPK190/0. The expression profiles of 13 genes (Table 1 ) were determined for all time-points in 1 quantitative experiment (see Supplemental material with Online version of paper). The in vitro differentiation process of the promastigotes could be synchronized for the two strains based on the concomitantly determined growth curve. The first day of stationary phase served as a reference point and was arbitrarily designated as time-point $0 \mathrm{~h}$. The matching expression profiles of the 24-hourly harvests could then be translated into synchronized 'expression-curves'. These expression-curves reflect the gene expression variation during differentiation over 8 days, and the type of profiles obtained for the 13 genes are once more exemplified by AQP1, GCS and TDR1. (i) Expression of AQP1 was relatively stable during the multiplicative phase, but rose sharply during the stationary phase with \pm 3 -fold difference between first $(0 \mathrm{~h})$ and second $(24 \mathrm{~h})$ day (Fig. 3A). This finding explains the variation between Experiments $\mathrm{A}$ and $\mathrm{B}$, which both investigated stationary phase promastigotes in the absence of synchronization. (ii) The profile of GCS was similar to AQP1, but the expression increase was much less pronounced ( $\pm 1 \cdot 5$-fold difference between 0 and $24 \mathrm{~h}$ ) (Fig. 3B). (iii) The last gene, TDR1, showed little variation 


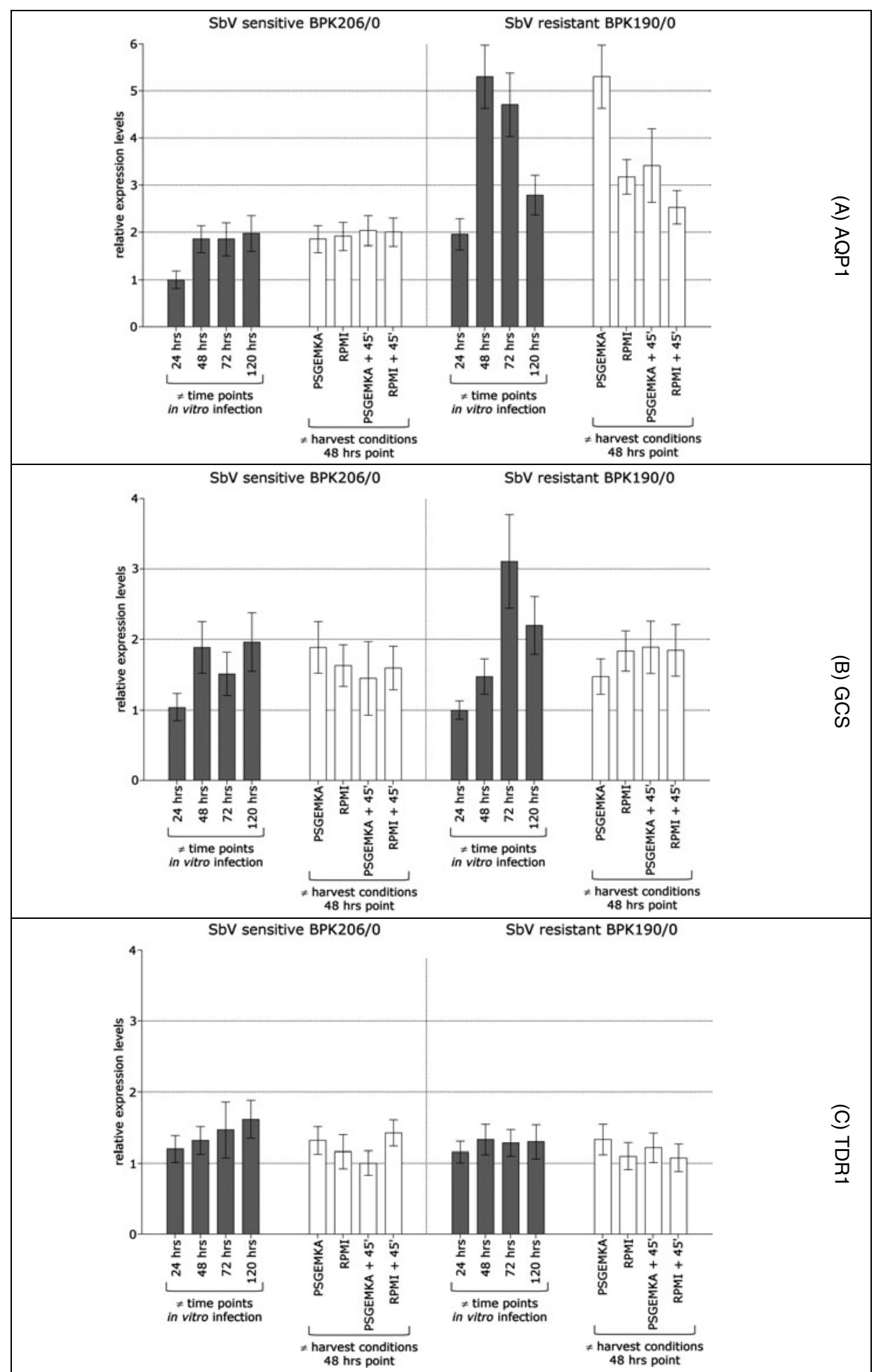

Fig. 2. Summary of expression profiling of Leishmania (L.) donovani intracellular amastigotes of the isolates BPK206/0 (SbV sensitive) and BPK190/0 (SbV resistant). Relative gene expression levels determined in variable biological conditions ( $\neq$ time-points of $i n$ vitro macrophage infections, grey bars) and using variable techniques $(\neq$ harvest conditions at time point $48 \mathrm{~h}$, white bars). A single large-scale macrophage infection with each isolate was used to test all conditions shown; expression levels were rescaled/gene versus sample with lowest expression, error bars = S.D.

over the whole promastigote differentiation process (Fig. 3C), this is also in agreement with Experiment A/B.

Just as with amastigotes, the degree of biological variation of expression during in vitro differentiation varies between different strains; this is clearly shown by the significant difference between BPK190/0 and BPK206/0 for AQP1.

The expression-curves of the 2 strains could be reproduced in a biological repeated experiment 


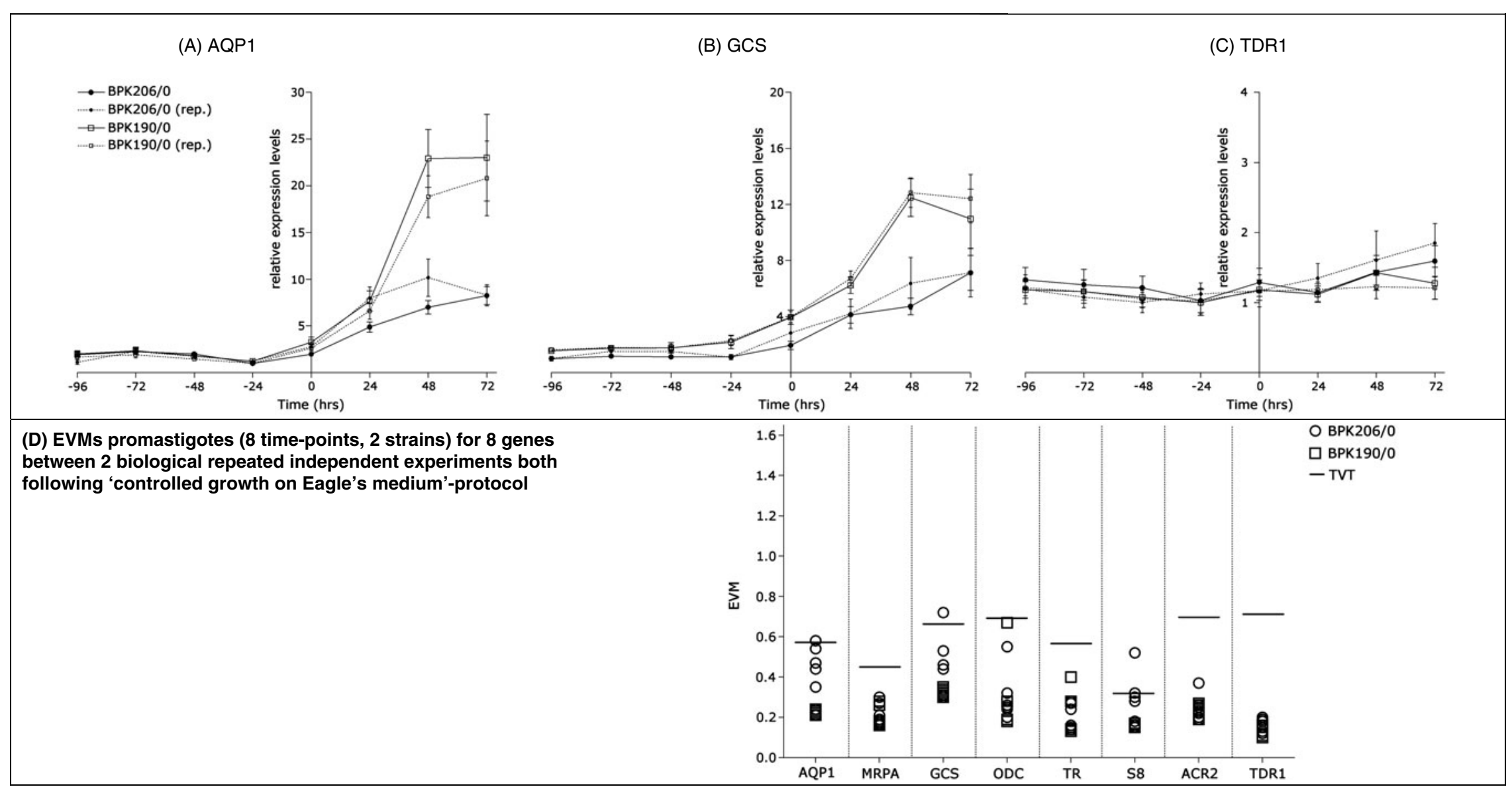

Fig. 3. Summary of expression profiling of Leishmania (L.) donovani promastigotes of the isolates BPK206/0 (SbV sensitive) and BPK190/0 (SbV resistant). (A-C) Expressioncurves reflecting biological variation in gene expression throughout in vitro promastigote differentiation; full lines and dotted lines (rep.) represent 2 biological repeated independent experiments. Total time-scale of 8 days with $0 \mathrm{~h}=1$ st day stationary phase according to concomitant growth curve; all expression levels/gene were rescaled versus sample with lowest expression in the respective experiment, error bars $=$ S.D. 
(dotted curves in Fig. 3A-C), even for AQP1. The 2 repeated independent experiments were scrutinized for inter-experimental biological variation by determining the EVM for 128 samples ( 8 timepoints $\times 2$ strains $\times 8$ genes), analogous to experiment A/B. We only identified 2 out of 128 samples with an EVM $>$ TVT (versus $7 / 32$ for promastigotes Experiment $\mathrm{A} / \mathrm{B}$ ) and there were no indications of consistent variation problems in any of the 8 genes (Fig. 3D).

\section{DISCUSSION}

Biochemical and molecular characterization of intracellular amastigotes has always been delicate, as purification of amastigotes from macrophages is usually required. Different methods of amastigote purification were reported in the past (Hart et al. 1981 ; Monjour et al. 1984; Pham and Mauel, 1987), but all involve long procedures $(>1 \mathrm{~h}$ ) and expose the amastigotes to chemical or physical strain. Hence, these techniques might influence the biological status (including gene expression profiles) of freed amastigotes as it is well-established that changes in environment rapidly $(<1 \mathrm{~h})$ trigger transformation to promastigotes (Fong and Chang, 1981; Duncan et al. 2001 ; Barak et al. 2005).

In this study, we used gene expression profiling for molecular characterization of intracellular amastigotes and introduced a new harvest-technique that omits the purification step (co-harvest amastigotes and macrophages). It was demonstrated, that even in the absence of amastigote purification, other technical parameters of the harvest procedure (e.g. type of buffer, time-span harvest) can still affect the expression profiles of co-harvested intracellular amastigotes (observed for 6 out of 13 tested genes). This was clearly exemplified by the unstable expression profile of the gene AQP1, which encodes a membrane channel thought to be involved in regulation of osmotic stress (Beitz, 2005). Possibly the expression profile of AQP1 continuously adjusts during in vitro manipulations in response to minor changes in medium/buffer, temperature, $\mathrm{pH}$, etc. Such a 'sensory' regulation could impede reproducible AQP1 expression profiling of amastigotes regardless of the technique applied for harvest.

We also established that expression profiles of in vitro generated intracellular amastigotes fluctuate significantly during the first $120 \mathrm{~h}$ post-infection (observed for 9 out of 13 genes). We suspect that this expression variation is, at least in part, associated with the ongoing transformation from promastigotes to amastigotes in those 5 days post-infection. A recent study on $L$. (L.) donovani reported similar findings during the transformation of promastigotes to axenic amastigotes (Saxena et al. 2007). The rate of this transformation process could vary between different isolates, thus there is no guarantee that various isolates reach identical transformation stages at any particular time-point in the first $120 \mathrm{~h}$ post-infection. A reliable molecular comparison of intracellular amastigotes should compare identical developmental stages and it seems therefore advisable to compare multiple time-points (in the form of expression-curves, as done here for promastigotes) in stead of 1 time-point.

These findings imply that comparison of results of various Leishmania amastigote gene expression studies needs to be done with extreme care if the studies use different in vitro manipulation protocols. For example, our previous molecular report on $L$. (L.) donovani $\mathrm{SbV}$ resistance was based on Experiment $\mathrm{A}$ and suggested that reduced expression of AQP1 and GCS in amastigotes was possibly a specific feature of $\mathrm{SbV}$-resistant isolates (Exp. A, Fig. 1) (Decuypere et al. 2005). However, now we obtained different results in the independent Experiment B, which differed in in vitro manipulation protocol. We could argue that the results could not be reproduced due to changes in the isolates (uncloned material) possibly introduced during culturing between Experiments A and B. However, in this particular example, it seems more likely that the amastigote purification step present in Experiment A but absent in Experiment $\mathrm{B}$, is (partly) responsible for the differences in amastigote expression profiles between Experiments A and B.

Characterization of promastigotes is less challenging; these extracellular parasites can easily be cultured and harvested with minimal risk of affecting their biological status. Gene expression profiles could be reproduced with the experimental procedures used here. However, as in the amastigote model, expression levels may vary throughout the in vitro differentiation from procyclic to metacyclic form (observed for 9 out of 13 tested genes). Changing gene expression profiles during promastigote differentiation has been reported before and this phenomenon is believed to be part of the molecular changes that take place to pre-adapt the parasite for transmission and survival in the vertebrate host (Sacks, 1989; Saxena et al. 2003; Akopyants et al. 2004; Almeida et al. 2004; CohenFreue et al. 2007). The in vitro differentiation rates of recently isolated parasites can differ significantly (unpublished observations), and thus it seems advisable to study expression-curves rather than single-point measurements. Expression-curves also confer the robustness required for expression profiling Leishmania, where, as discussed before, differences in expression level are often much lower than in other eukaryotes (Akopyants et al. 2004; Almeida et al. 2004; Duncan, 2004). While one particular measurement may not be significantly different between different strains; the picture of multiple measurements can reveal a significant differential expression regulation as is seen with 
the genes AQP1 and GCS for the 2 strains studied here.

Gene expression studies on promastigotes cannot replace expression studies on intracellular amastigotes, as the latter provides unique information on the clinical relevant form of the parasite at the time of interaction with the host-cell. However, promastigote expression-curves can provide complementary information on the cell biological flexibility of the parasite. More specifically, the promastigote expression-curves capture the degree of mRNA abundance regulation during in vitro differentiation in a standardized environment and as such reflect the characteristic adaptive capacity of an isolate. The expression-curves of the two isolates tested here suggest that different isolates can present with a differential degree of regulation (adaptive capacity) which in turn might be linked to differential phenotypes.

In their natural context, Leishmania are characterized by a tremendous phenotypic diversity regarding vector-specificity, virulence, drug susceptibility etc. This diversity contrasts with the relative structural conservation of Leishmania genomes (Peacock et al. 2007). Possibly, Leishmania's natural phenotypic diversity lies in differential features downstream of the genome, in the transcriptome or in the proteome. Consequently, gene expression profiling could play an essential role in the characterization of phenotypic distinct Leishmania isolates. This study demonstrated that highly standardized methods are required for reliable comparative analyses. We believe it is particularly essential to adopt methods that conserve the environment of the parasite and take into account the fluctuation of gene expression levels during the life-cycle of the parasite. Following these guidelines, a profiling assay was developed for promastigotes, but further studies are still required to achieve a similar highly standardized method for intracellular amastigotes.

This work was supported by the Fifth Framework Programme of the European Commission Community Research (INCO-DEV contract LeishNatDrug-R - ICACT-2001-10076), the Sixth Framework Programme of the European Commission Community Research (INCODEV contract LeishEpiNet - INCO-CT2005-015407 and Marie Curie IEF contract DonNA-R - 041465) and the Research Foundation Flanders (FWO) (project 6.0103.06). We thank Gareth Westrop for critically reading this manuscript.

\section{REFERENCES}

Akopyants, N. S., Matlib, R. S., Bukanova, E. N., Smeds, M. R., Brownstein, B. H., Stormo, G. D. and Beverley, S. M. (2004). Expression profiling using random genomic DNA microarrays identifies differentially expressed genes associated with three major developmental stages of the protozoan parasite Leishmania major. Molecular and Biochemical Parasitology 136, 71-86.
Almeida, R., Gilmartin, B. J., McCann, S. H., Norrish, A., Ivens, A. C., Lawson, D., Levick, M. P., Smith, D. F., Dyall, S. D., Vetrie, D., Freeman, T. C., Coulson, R. M. and others (2004). Expression profiling of the Leishmania life cycle: cDNA arrays identify developmentally regulated genes present but not annotated in the genome. Molecular and Biochemical Parasitology 136, 87-100.

Barak, E., Amin-Spector, S., Gerliak, E., Goyard, S., Holland, N. and Zilberstein, D. (2005).

Differentiation of Leishmania donovani in host-free system: analysis of signal perception and response. Molecular and Biochemical Parasitology 141, 99-108.

Bates, P. A. and Tetley, L. (1993). Leishmania mexicana: induction of metacyclogenesis by cultivation of promastigotes at acidic $\mathrm{pH}$. Experimental Parasitology 76, 412-423.

Beitz, E. (2005). Aquaporins from pathogenic protozoan parasites: structure, function and potential for chemotherapy. Biology of the Cell 97, 373-383.

Boucher, N., Wu, Y., Dumas, C., Dube, M., Sereno, D., Breton, M. and Papadopoulou, B. (2002). A common mechanism of stage-regulated gene expression in Leishmania mediated by a conserved 3'-untranslated region element. The Fournal of Biological Chemistry 277, 19511-19520.

Campbell, D. A., Thomas, S. and Sturm, N. R. (2003). Transcription in kinetoplastid protozoa: why be normal? Microbes and Infection 5, 1231-1240.

Chaussabel, D., Semnani, R. T., McDowell, M. A., Sacks, D., Sher, A. and Nutman, T. B. (2003). Unique gene expression profiles of human macrophages and dendritic cells to phylogenetically distinct parasites. Blood 102, 672-681.

Clayton, C. E. (2002). Life without transcriptional control? From fly to man and back again. The EMBO Fournal 21, 1881-1888.

Cohen-Freue, G., Holzer, T. R., Forney, J. D. and McMaster, W. R. (2007). Global gene expression in Leishmania. International Fournal for Parasitology 37, 1077-1086.

Decuypere, S., Rijal, S., Yardley, V., De Doncker, S., Laurent, T., Khanal, B., Chappuis, F. and Dujardin, J. C. (2005). Gene expression analysis of the mechanism of natural $\mathrm{Sb}(\mathrm{V})$ resistance in Leishmania donovani isolates from Nepal. Antimicrobial Agents and Chemotherapy 49, 4616-4621.

Duncan, R. (2004). DNA microarray analysis of protozoan parasite gene expression: outcomes correlate with mechanisms of regulation. Trends in Parasitology 20, 211-215.

Duncan, R., Alvarez, R., Jaffe, C. L., Wiese, M., Klutch, M., Shakarian, A., Dwyer, D. and Nakhasi, H. L. (2001). Early response gene expression during differentiation of cultured Leishmania donovani. Parasitology Research 87, 897-906.

Duncan, R. C., Salotra, P., Goyal, N., Akopyants, N. S., Beverley, S. M. and Nakhasi, H. L. (2004). The application of gene expression microarray technology to kinetoplastid research. Current Molecular Medicine 4, 611-621.

Fong, D. and Chang, K. P. (1981). Tubulin biosynthesis in the developmental cycle of a parasitic protozoan, Leishmania mexicana: changes during differentiation 
of motile and nonmotile stages. Proceedings of the

National Academy of Sciences, USA 78, 7624-7628.

Hart, D. T., Vickerman, K. and Coombs, G. H. (1981). A quick, simple method for purifying Leishmania mexicana amastigotes in large numbers. Parasitology 82, 345-355.

Heid, C. A., Stevens, J., Livak, K. J. and Williams, P. M. (1996). Real time quantitative PCR. Genome Research 6, 986-994.

Hellemans, J., Mortier, G., De Paepe, A., Speleman, F. and Vandesompele, J. (2007). qBase relative quantification framework and software for management and automated analysis of real-time quantitative PCR data. Genome Biology 8, R19.

Holzer, T. R., McMaster, W. R. and Forney, J. D. (2006). Expression profiling by whole-genome interspecies microarray hybridization reveals differential gene expression in procyclic promastigotes, lesion-derived amastigotes, and axenic amastigotes in Leishmania mexicana. Molecular and Biochemical Parasitology 146, 198-218.

Hromatka, B. S., Noble, S. M. and Johnson, A. D. (2005). Transcriptional response of Candida albicans to nitric oxide and the role of the YHB1 gene in nitrosative stress and virulence. Molecular Biology of the Cell 16, 4814-4826.

Ivens, A. C., Peacock, C. S., Worthey, E. A., Murphy, L., Aggarwal, G., Berriman, M., Sisk, E., Rajandream, M. A., Adlem, E., Aert, R., Anupama, A., Apostolou, Z. and others (2005). The genome of the kinetoplastid parasite, Leishmania major. Science 309, 436-442.

Laurent, T., Rijal, S., Yardley, V., Croft, S., De Doncker, S., Decuypere, S., Khanal, B., Singh, R., Schonian, G., Kuhls, K., Chappuis, F. and Dujardin, J. C. (2007). Epidemiological dynamics of antimonial resistance in Leishmania donovani: Genotyping reveals a polyclonal population structure among naturallyresistant clinical isolates from Nepal. Infection, Genetics and Evolution 7, 206-212.

Martinez-Calvillo, S., Nguyen, D., Stuart, K. and Myler, P. J. (2004). Transcription initiation and termination on Leishmania major chromosome 3. Eukaryotic Cell 3, 506-517.

McAleese, F., Wu, S. W., Sieradzki, K., Dunman, P., Murphy, E., Projan, S. and Tomasz, A. (2006). Overexpression of genes of the cell wall stimulon in clinical isolates of Staphylococcus aureus exhibiting vancomycin-intermediate-S. aureus-type resistance to vancomycin. Fournal of Bacteriology 188, 1120-1133.

McNicoll, F., Drummelsmith, J., Muller, M., Madore, E., Boilard, N., Ouellette, M. and Papadopoulou, B. (2006). A combined proteomic and transcriptomic approach to the study of stage differentiation in Leishmania infantum. Proteomics 6 , 3567-3581.

Monjour, L., Vouldoukis, I., Brandicourt, O., Mazier, D., Alfred, C., Ploton, I. and Gentilini, M. (1984). Rapid, large-scale production and isolation for Leishmania amastigotes. Annals of Tropical Medicine and Parasitology 78, 423-425.

Mottram, J. C., Robertson, C. D., Coombs, G. H. and Barry, J. D. (1992). A developmentally regulated cysteine proteinase gene of Leishmania mexicana. Molecular Microbiology 6, 1925-1932.

Peacock, C. S., Seeger, K., Harris, D., Murphy, L., Ruiz, J. C., Quail, M. A., Peters, N., Adlem, E., Tivey, A., Aslett, M., Kerhornou, A., Ivens, A. and others (2007). Comparative genomic analysis of three Leishmania species that cause diverse human disease. Nature Genetics 39, 839-847.

Pham, T. V. and Mauel, J. (1987). Studies on intracellular killing of Leishmania major and lysis of host macrophages by immune lymphoid cells in vitro. Parasite Immunology 9, 721-736.

Rijal, S., Yardley, V., Chappuis, F., Decuypere, S., Khanal, B., Singh, R., Boelaert, M., De Doncker, S., Croft, S. and Dujardin, J. C. (2007). Antimonial treatment of visceral leishmaniasis: are current in vitro susceptibility assays adequate for prognosis of in vivo therapy outcome? Microbes and Infection $\mathbf{9}$, 529-535.

Sacks, D. L. (1989). Metacyclogenesis in Leishmania promastigotes. Experimental Parasitology 69, 100-103.

Saxena, A., Lahav, T., Holland, N., Aggarwal, G., Anupama, A., Huang, Y., Volpin, H., Myler, P. J. and Zilberstein, D. (2007). Analysis of the Leishmania donovani transcriptome reveals an ordered progression of transient and permanent changes in gene expression during differentiation. Molecular and Biochemical Parasitology 152, 53-65.

Saxena, A., Worthey, E. A., Yan, S., Leland, A., Stuart, K. D. and Myler, P. J. (2003). Evaluation of differential gene expression in Leishmania major Friedlin procyclics and metacyclics using DNA microarray analysis. Molecular and Biochemical Parasitology 129, 103-114.

Schena, M., Shalon, D., Davis, R. W. and Brown, P. O. (1995). Quantitative monitoring of gene expression patterns with a complementary DNA microarray. Science 270, 467-470.

Shapira, M., McEwen, J. G. and Jaffe, C. L. (1988). Temperature effects on molecular processes which lead to stage differentiation in Leishmania. The EMBO Fournal 7, 2895-2901.

Tintaya, K. W., Ying, X., Dedet, J. P., Rijal, S., De Bolle, X. and Dujardin, J. C. (2004). Antigen genes for molecular epidemiology of leishmaniasis: polymorphism of cysteine proteinase $\mathrm{B}$ and surface metalloprotease glycoprotein 63 in the Leishmania donovani complex. The Yournal of Infectious Diseases 189 1035-1043.

Tobie, E. J., Von Brand, T. and Mehlman, B. (1950). Cultural and physiological observations on Trypanosoma rhodesiense and Trypanosoma gambiense. Fournal of Parasitology 36, 48-54.

Vandesompele, J., De Preter, K., Pattyn, F., Poppe, B., Van Roy, N., De Paepe, A. and Speleman, F. (2002). Accurate normalization of real-time quantitative RT-PCR data by geometric averaging of multiple internal control genes. Genome Biology 3, research 0034.1-0034.11.

Zakai, H. A., Chance, M. L. and Bates, P. A. (1998). In vitro stimulation of metacyclogenesis in Leishmania braziliensis, L. donovani, L. major and L. mexicana. Parasitology 116, 305-309. 